EIVIRA FERRERA MARQUES ${ }^{1}$

Julana Alves SOUZA ${ }^{2}$

LUCIANA GRAZIANO ${ }^{3}$

Almir Galvão Vieira Bitencourt ${ }^{4}$

Cristiano Senaga ${ }^{5}$

Carlos Eduardo Martins Fontes ${ }^{6}$

\section{Músculo esternal simulando nódulo mamário}

\author{
Sternalis muscle simulating a breast nodule
}

Artigo original

Palavras-chave

Doenças mamárias/diagnóstico

Neoplasias mamárias/diagnóstico

Mamografia

Tomografia computadorizada por raios $X$ Imagem por ressonância magnética

Diagnóstico diferencial

Parede torácica

Músculos

Keywords

Breast deseases/diagnosis Breast neoplasms/diagnosis Mammography Tomography, X-ray computed Magnetic resonance imaging

Diagnosis, differentia

Thoracic wall

Muscles

\section{Resumo}

OBJETIVO: relatar uma série de três casos de uma normal variation conhecida como músculo esternal simulando lesão mamária. MÉTODOS: a suspeita diagnóstica foi feita a partir do quadro clínico, achados no exame físico e aspectos de imagem, sendo confirmada por meio de métodos de imagem seccionais, como tomografia computadorizada (TC) e ressonância magnética (RM). Foi realizada uma revisão da literatura no banco de dados Medline e em livros-texto de radiologia mamária sobre os aspectos anatômicos, clínicos e de imagem do músculo esternal. RESULTADOS: três pacientes do sexo feminino, sem queixas, que apresentaram lesões mamárias de aspecto nodular na projeção dos quadrantes mediais (duas em exame mamográfico de rotina e uma em TC). $\bigcirc$ diagnóstico de músculo esternal foi confirmado por meio de RM de mamas ou TC de tórax, demonstrando imagem alongada, localizada na região paraesternal esquerda, adjacente ao músculo peitoral. CONCLUSÕES: o músculo esternal é uma variante não usual da musculatura da parede torácica, presente em cerca de 2 a $8 \%$ da população. $O$ conhecimento dessa entidade é fundamental, pois pode simular nódulo mamário.

\section{Abstract}

PURPOSE: to report a series of three cases of a normal variation known as sternal muscle, simulating a breast lesion. METHODS: the diagnostic suspicion was based on the clinical picture, findings in the physical examination and imaging, being confirmed by sectional imaging methods such as computerized tomography (CT) and magnetic resonance imaging (MRI). A review of the literature has been made in the data Medline and in breast radiology textbooks about the anatomic, clinical and imaging aspects of the sternal muscle. RESULTS: three female patients, without complaints, who presented nodular breast lesions in the medial quadrants projection (two in a routine mammographic exam and one in a computerized tomography). The diagnosis of sternal muscle was confirmed through breast MRI or through thoracic $\mathrm{CT}$, showing an elongated image in the left parasternal region, adjacent to the breast muscle. CONCLUSIONS: the sternal muscle is an unusual variation of the muscles of the thoracic wall, present in about 2 to $8 \%$ of the population. The knowledge of this entity is crucial, as it can simulate a breast node. CEP 01509-010 - São Paulo (SP), Brasi Fones: (11) 2189-5000 ramal 1066 E-mail: anluciana@ig.com.br

Recebido

21/7/09

\section{Departamento de Imagem do Hospital A. C. Camargo - São Paulo (SP), Brasil.}

'Médico Titular do Setor de Mamografia do Departamento de Imagem do Hospital A. C. Camargo - São Paulo (SP), Brasil.

${ }^{2}$ Médico Titular do Setor de Mamografia do Departamento de Imagem do Hospital A. C. Camargo - São Paulo (SP), Brasil.

${ }^{3}$ Residente de Radiologia do Diagnóstico por Imagem do Hospital A. C. Camargo - São Paulo (SP), Brasil.

${ }^{4}$ Residente de Radiologia do Diagnóstico por Imagem do Hospital A. C. Camargo - São Paulo (SP), Brasil.

${ }^{5}$ Residente de Radiologia do Diagnóstico por Imagem do Hospital A. C. Camargo - São Paulo (SP), Brasil.

- Médico Titular do Departamento de Mastologia do Hospital A. C. Camargo - São Paulo (SP), Brasil. 


\section{Introdução}

A mama é um órgão peculiar, pois não apresenta um padrão universal. Entretanto, certos achados são característicos, e a identificação destes durante os exames de imagem permite uma avaliação adequada. Para isso, é preciso conhecer os padrões de mama e fazer a avaliação, quando possível, comparativamente ${ }^{1}$. O reconhecimento das variações da normalidade (por exemplo, mama acessória) e pseudolesões (por exemplo, lóbulo de tecido adiposo) é imprescindível, pois, um diagnóstico preciso evita procedimentos inadequados e desnecessários ${ }^{2}$.

Dentre as variantes anatômicas, destacam-se as variações da musculatura da parede torácica. A inserção medial do músculo peitoral maior é variável e em cerca de $1 \%$ das mulheres pode ser visualizado em mamografias, na projeção medial na incidência crânio-caudal $(\mathrm{CC})^{3}$. Neste contexto, essa estrutura pode assumir uma conformação arredondada, triangular ou em "chama de vela", simulando uma lesão maligna ${ }^{4}$.

Além das variações na inserção do músculo peitoral, a presença de uma estrutura muscular acessória, denominada músculo esternal, também pode ser confundida com tumor na mamografias. O objetivo deste trabalho foi relatar três casos que apresentavam o músculo esternal simulando lesões mamárias.

\section{Métodos}

Nesse artigo, são descritos os casos de três pacientes encaminhadas ao Departamento de Imagem do Hospital A. C. Camargo com suspeita de nódulo/ massa na projeção dos quadrantes mediais da mama. A suspeita diagnóstica foi feita a partir do quadro clínico, achados no exame físico e aspectos de imagem, sendo confirmada por meio de métodos de imagem seccionais, como tomografia computadorizada (TC) e ressonância magnética (RM).

Foi realizada uma revisão da literatura, no banco de dados Medline e em livros-texto de radiologia mamária, sobre os aspectos anatômicos, clínicos e de imagem do músculo esternal, bem como de outras variações anatômicas e pseudolesões simulando nódulos mamários na mamografia.

\section{Resultados}

\section{Caso 1}

Paciente do sexo feminino, 40 anos, sem queixas ou alterações ao exame físico. Realizou exame mamográfico de rotina, que evidenciou assimetria visualizada somente na incidência CC, na projeção dos quadrantes mediais da mama esquerda, em região profunda, com atenuação radiológica finamente heterogênea, com áreas de baixa densidade de permeio, que persistia na incidência em clivagem (Figura 1). Esta lesão foi classificada na categoria 0 do sistema Breast Imaging Report and Data System (BI-RADSTM), sendo sugerida a avaliação complementar com ultrassonografia (US) ou RM. Posteriormente, foi realizada US, que não demonstrou alterações, e RM das mamas, evidenciando imagem alongada, localizada na região paraesternal esquerda, com o mesmo sinal da musculatura peitoral nas sequências em T1, T2 e póscontraste (Figura 2). O diagnóstico foi concluído como músculo esternal.

\section{Caso 2}

Paciente do sexo feminino, 46 anos, durante exame mamográfico de rotina, apresentou imagem nodular na projeção dos quadrantes mediais da mama direita, somente visualizada na incidência CC (Figuras 3A e

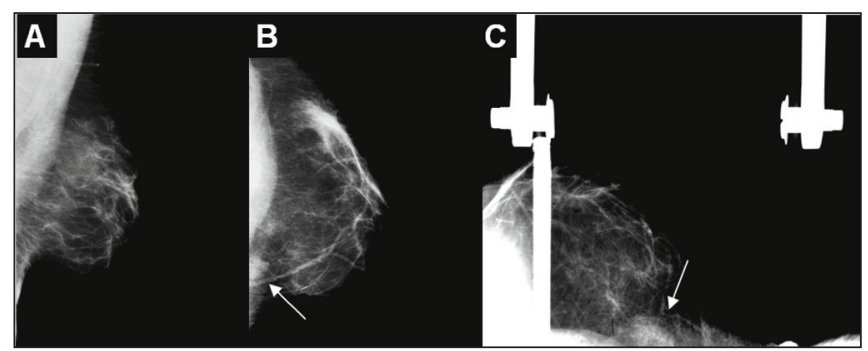

Figura 1 - Mamografia esquerda sem evidência de alterações na incidência médio-lateral-óbliqua $(A)$, demonstrando área com aspecto nodular na região profunda dos quadrantes mediais (seta) na incidência crânio-caudal (B), melhor visualizada na incidência em clivagem com compressão localizada (C).

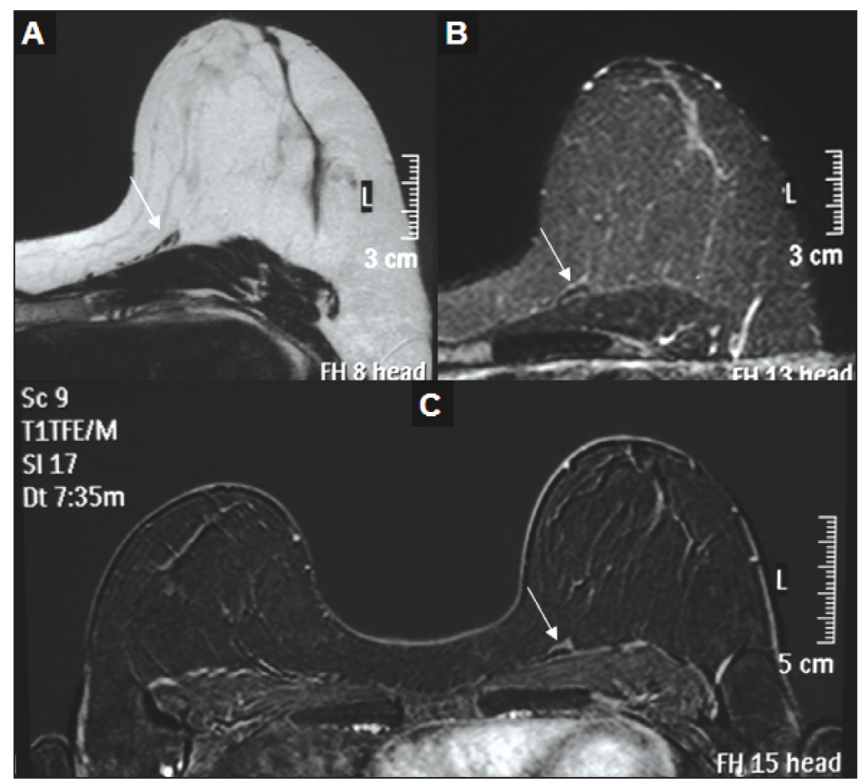

Figura 2 - Cortes axiais de ressonância magnética nas sequências em T1 sem contraste (A), T2 (B) e T1 pós-contraste com saturação de gordura (C), demonstrando músculo esternal (setas) como imagem adjacente ao músculo peitoral, localizada na região paraesternal da mama esquerda, com o mesmo sinal da musculatura em todas sequências. 


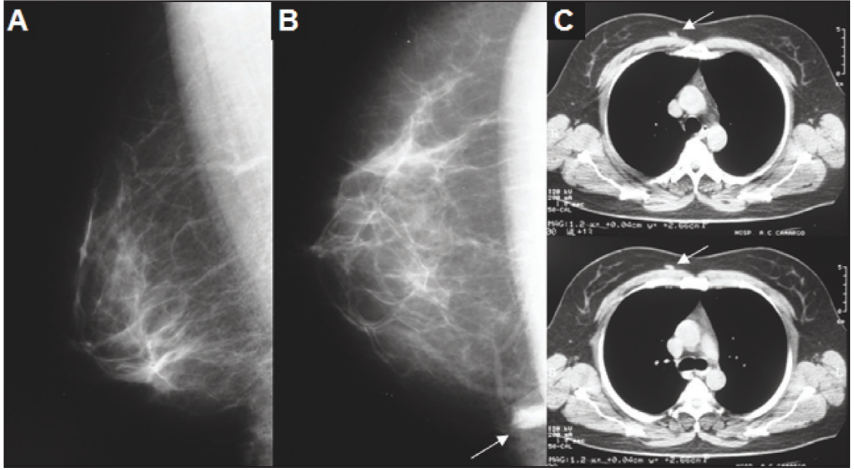

Figura 3 - Mamografia direita sem evidência de alterações na incidência médio-lateral-óbliqua (A), demonstrando área com aspecto nodular na região profunda dos quadrantes mediais (seta) na incidência crânio-caudal (B). Cortes axiais de tomografia computadorizada (C), janela de partes moles, demonstram músculo esternal como imagem adjacente ao músculo peitoral direito, localizada ao longo da região paraesternal (setas).

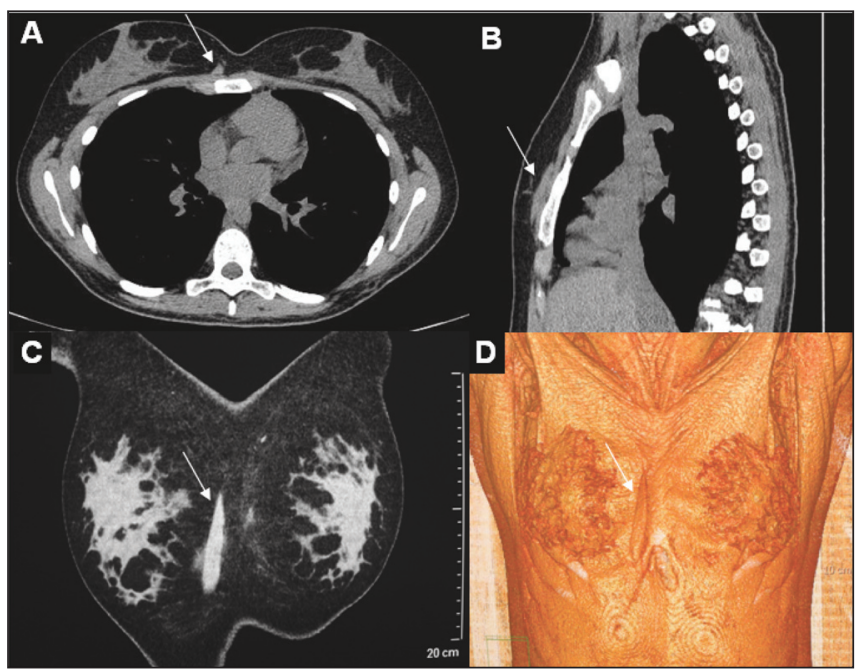

Figura 4 - Tomografia computadorizada de tórax evidenciando músculo esternal à direita (setas): corte axial $(A)$, reconstruções em sagital $(B)$ e coronal (C), e reconstrução 3D com Volume Rendering (D).

3B). O diagnóstico de músculo esternal foi suspeitado e confirmado por TC de tórax, que evidenciou imagem alongada na região paraesternal direita, adjacente ao músculo peitoral (Figura 3C).

\section{Caso 3}

Paciente do sexo feminino, 17 anos, sem queixas, realizou TC de tórax como seguimento de leucemia tratada, na qual foi descrita imagem nodular no quadrante ínfero-medial na mama direita (imagens não disponíveis). A paciente foi submetida à US das mamas e a exame mamográfico, que não demonstraram alterações. Para melhor avaliação, foi solicitada nova TC de tórax, em equipamento com múltiplos detectores, e realizadas reconstruções nos planos sagital e coronal, e reconstrução em 3D, na qual a lesão previamente descrita foi caracterizada como músculo esternal (Figura 4).

\section{Discussão}

O músculo esternal é uma variante não usual da musculatura da parede torácica presente em 2 a $8 \%$ da população geral ${ }^{6,7}$. Pode ser um achado uni ou bilateral, apresentando-se como uma estrutura alongada na região paraesternal, adjacente ao músculo peitoral maior. Na mamografia, o achado típico é de uma densidade arredondada ou irregular, na maioria das vezes rodeada por tecido adiposo, visualizada na projeção medial profunda da mama na incidência CC, medindo cerca de 1 a $2 \mathrm{~cm}^{5,8}$. Caracteristicamente, essa estrutura não é visualizada na incidência Médio lateral obliqua (MLO). Acredita-se que ele apareça nas imagens mamográficas quando o músculo está relaxado ${ }^{8}$.

O músculo esternal foi primeiramente descrito em 1604, por Cabrolius, no entanto, até hoje, a origem dessa rara normal variation ainda é tema de discussão9. Algumas etiologias são propostas, mas uma das mais aceitas é a de que o músculo esternal tem suas fibras em situação crânio-caudal, na bainha do músculo reto abdominal ou estruturas adjacentes, terminando sobre a fáscia peitoral, acima do esterno, cartilagem costal ou na cabeça medial do músculo esternocleidomastoideo ${ }^{5,10}$. Não se sabe, ao certo, a função deste músculo.

A presença deste músculo extranumerário é descrita como uma entidade não familiarizada pelos cirurgiões que atuam na área de mastologia. O conhecimento do músculo esternal é relevante no ato cirúrgico para identificação dos planos anatômicos durante uma cirurgia mamária ${ }^{11,12}$. Uma vez identificado e separado, é recomendada uma nota cirúrgica para posterior seguimento, para que sejam evitadas falsas interpretações e ou causadas novas ambiguidades ${ }^{13}$.

O diagnóstico é definido pela típica localização e configuração na mamografia, com ausência de anormalidades correspondentes nas incidências laterais, além de exame físico normal. A US pode demonstrar a presença do músculo esternal como uma estrutura paraesternal alongada com ecogenicidade igual ou semelhante ao músculo peitoral adjacente ${ }^{14}$. No entanto, este pode passar despercebido se o exame não for direcionado para este fim.

Conforme demonstrado nos casos apresentados, para melhor caracterização dessa variante da normalidade, podem ser realizadas RM de mamas ou ainda TC de tórax com múltiplos detectores, a qual permite reconstruções multiplanares ${ }^{14}$. $\mathrm{Na}$ atualidade, o exame de RM é considerado mais completo, pois, além de caracterizar o músculo esternal, ajuda a excluir possíveis lesões mamárias malignas por meio de sequências específicas e avaliação do estudo dinâmico ${ }^{10}$.

Portanto, os mastologias, cirurgiões plásticos e radiologistas especializados em imagem da mama devem ter conhecimento dessa entidade, para reduzir a necessidade de exames adicionais e evitar a indicação de procedimentos desnecessários. 


\section{Referências}

1. Colégio Brasileiro de Radiologia. BI-RADS: Sistema de Laudos e Registro de Dados de Imagem da Mama. São Paulo: Colégio Brasileiro de Radiologia; 2005.

2. Pojchamarnwiputh $S$, Muttarak M, Na-Chiangmai W, Chaiwun B. Benign breast lesions mimicking carcinoma at mammography. Singapore Med J. 2007;48(10):958-68.

3. Kopans DB. Breast anatomy and basic histology, physiology, and pathology. In: Kopans DB. Breast imaging. 3rd ed. Philadelphia: Lippincott Williams \& Wilkins; 2007. chap. 2.

4. Cawson JN, Papadopoulos T. Variants of sternal insertion of the pectoral muscle on mammography: a pictorial review. Clin Radiol. 2002; 57(6):442-8.

5. Bradley FM, Hoover HC Jr, Hulka CA, Whitman GJ, McCarthy KA, Hall DA, et al. The sternalis muscle: an unusual normal finding seen on mammography. AJR Am J Roentgenol. 1996;166(1):33-6.

6. Scott-Conner CE, Al-Jurf AS. The sternalis muscle. Clin Anat. 2002;15(1):67-9.

7. Rahman NA, Das S, Maatoq Sulaiman I, Hlaing KP, Haji Suhaimi $\mathrm{F}$, Latiff $\mathrm{AA}$, et al. The sternalis muscle in cadavers: anatomical facts and clinical significance. Clin Ter. 2009;160(2):129-31.
8. Young Lee B, Young Byun J, Hee Kim H, Sook Kim H, Mee Cho S, Hoon Lee K, et al. The sternalis muscles: incidence and imaging findings on MDCT. J Thorac Imaging. 2006;21 (3):179-83.

9. Novakov SS, Yotova NI, Petleshkova TD, Muletarov SM. Sternalis muscle-a riddle that still awaits an answer short communication. Folia Med (Plovdiv). 2008;50(2):63-6.

10. Goktan C, Orguc S, Serter S, Ovali GY. Musculus sternalis: a normal but rare mammographic finding and magnetic resonance imaging demonstration. Breast J. 2006;12(5):488-9.

11. Bailey PM, Tzarnas CD. The sternalis muscle: a normal finding encountered during breast surgery. Plast Reconstr Surg. 1999; 103(4): 11 189-90.

12. Kabay B, Akdogan I, Ozdemir B, Adiguzel E. The left sternalis muscle variation detected during mastectomy. Folia Morphol (Warsz). 2005;64(4):338-40.

13. Harish K, Gopinath KS. Sternalis muscle: importance in surgery of the breast. Surg Radiol Anat. 2003;25(3-4):311-4.

14. Nuthakki S, Gross M, Fessell D. Sonography and helical computed tomography of the sternalis muscle. J Ultrasound Med. $2007 ; 26(2): 247-50$ 